\title{
RELATIONSHIP BETWEEN SOCIOECONOMIC FACTORS AND FNA RESULTS BY THE REGIONS OF BRAZIL
}

Amanda Cristina de Souza Vieira', Bianca Borges Martins¹, Matheus Nascimento Duarte', Thiago de Moura Arruda1', Wglaison Paulo Araújo Sobral', Fabiana Cândida de Queiroz Santos Anjos', Priscila Ferreira Barbosa 'Universidade de Gurupi - Gurupi (TO), Brazil.

Objective: The aim of this study was to analyze a prevalence relationship between the results of breast cytopathological examinations and the regions of Brazil. Methodology: This is a retrospective, epidemiological, and cross-sectional study, where the data were obtained from the Breast Cancer Information System, regarding the results of breast cytopathological examinations performed by fine-needle aspiration (FNA), from January 2009 to July 2015. The Brazilian North, Northeast, Southeast, South, and Midwest regions were selected. The FNA result variables selected were as follows: benign negative malignancy processes, compatible undetermined malignancy, suspect for malignancy, positive for malignancy, and inconsistent information. The collected data were tabulated and treated statistically to determine the absolute prevalence, analyzing the percentage relationship between the results of the FNA and social conditions of the regions. Results: In total, 63,240 cytopathologies were reported by FNA in Brazil, the region with the highest prevalence of this examination was the Southeast, with $39 \%$ ( $n=24,618)$, followed by the Northeast, with $30.3 \%(n=19,162)$, being the North, the lowest prevalence of notifications, $2.6 \%$ ( $\mathrm{n}=1,665)$. Among the results of the FNA, the highest frequency of the examinations resulted in a benign result, representing $88 \%$ of the total $(n=55,685)$. The highest incidence of biopsies positive for malignancy was in the Southeast, 8.3\% ( $\mathrm{n}=2,056)$. Conclusion: The Southeast has greater socioeconomic development, which contributes to an increase in risk factors for women in the region, such as a lower number of pregnancies, as well as postponing it. The results found leave room for further investigation, given that populous regions, such as the North, and with significant population aging, such as the Midwest and the South of Brazil, reported a very small number of biopsies performed, which may reflect failures in public health policy, difficulty in accessing the test or underreporting of this procedure.

Keywords: Biopsy; Needle; Risk Factors; Breast Neoplasms. 